\title{
Estabilización de suelos arcillosos a nivel de subrasante con adición de bolsas de polietileno fundido
}

\section{Stabilization of clay soils at the subgrade level with the addition of molten polyethylene bags}

\author{
Roiser Rene Linares Chavez, Manuel Eduardo Aguilar Rojas², Edward Enrique Rojas De La Puente
}

\section{RESUMEN}

La finalidad de la investigación fue determinar la influencia de las bolsas de polietileno fundido en la estabilización de un suelo arcilloso a nivel de subrasante, las muestras fueron extraídas de la intersección de la Av. Los Libertadores y Jr. Las Orquídeas, Pueblo Joven 16 de Octubre, Chachapoyas, Amazonas. Para elaborar la muestra, se fundieron bolsas de polietileno, el líquido producto de la fundición se mezcló con el material del suelo arcilloso en la proporción 1:1. El agregado base constituido, sirvió para elaborar las muestras tomando proporciones de 4\%, $8 \%$ y $12 \%$ respecto al peso seco de la muestra. El tratamiento que presentó mejor comportamiento para las propiedades físicas es el T1 (4\%) donde se logró una disminución promedio del índice de plasticidad de $13.55 \%$ (suelo natural) hasta $8.98 \%$ (4\% BPF). Y para las propiedades mecánicas el tratamiento que presenta mejor incidencia es el T3 (12\%) ya que el OCH promedio tiende a disminuir de $18.23 \%$ (suelo natural) a $15.46 \%(12 \%$ $\mathrm{BPF}$ ), mientras que la MDS promedio aumenta de $1.730 \mathrm{gr} / \mathrm{cm} 3$ (suelo natural) a $1.807 \mathrm{gr} / \mathrm{cm} 3(12 \% \mathrm{BPF})$ y el CBR promedio aumenta de 5.6\% (suelo natural) a 9.9\% (12\% BPF). Se concluye que la adición de bolsas de polietileno fundido si mejoran las propiedades físicas y mecánicas del suelo arcilloso a nivel de subrasante, por lo tanto, se logró estabilizar el suelo en estudio según del Manual de Carreteras Suelos, Geología, Geotecnia y Pavimentos.

Palabras clave: Estabilización de suelos, bolsas de polietileno.

\section{ABSTRACT}

The purpose of the research was to determine the influence of molten polyethylene bags in the stabilization of a clay soil at the subgrade level, the samples were taken from the intersection of Av. Los Libertadores and Jr. Las Orquídeas, Pueblo Joven 16 de October, Chachapoyas, Amazonas. To make the sample, polyethylene bags were melted, the melting product liquid was mixed with the clay soil material in the ratio 1: 1. The base aggregate constituted, served to prepare the samples taking proportions of $4 \%, 8 \%$ and $12 \%$ with respect to the dry weight of the sample. The treatment that presented the best performance for physical properties is T1 (4\%) where an average decrease in the plasticity index was achieved from $13.55 \%$ (natural soil) to $8.98 \%$ (4\% GMP). And for mechanical properties, the treatment with the best incidence is T3 (12\%) since the average OCH tends to decrease from $18.23 \%$ (natural soil) to $15.46 \%$ (12\% GMP), while the average MDS increases from 1,730. gr / cm3 (natural soil) to $1.807 \mathrm{gr} / \mathrm{cm} 3$ (12\% GMP) and the average CBR increases from 5.6\% (natural soil) to $9.9 \%$ (12\% GMP). It is concluded that the addition of molten polyethylene bags does improve the physical and mechanical properties of the clayey soil at the subgrade level, therefore, it was possible to stabilize the soil under study according to the Manual of Roads Soils, Geology, Geotechnics and Pavements.

Keywords: Soil stabilization, polyethylene bags.

\footnotetext{
Bachiller en Ingeniería Civil, Universidad Nacional Toribio Rodríguez de Mendoza de Amazonas.

${ }^{2}$ Ingeniero Civil, docente de la Universidad Nacional Toribio Rodríguez de Mendoza de Amazonas.

${ }^{3}$ Doctor, docente de la Universidad Nacional Toribio Rodríguez de Mendoza de Amazonas.
} 


\section{INTRODUCCIÓN}

La estabilización de suelos es el mejoramiento de las propiedades de un suelo a través de procedimientos mecánicos e incorporación de productos químicos, naturales y sintéticos. Tales estabilizaciones, por lo general, se realizan en los suelos de subrasante inadecuado o pobre, en este caso son conocidos como estabilización suelo cemento, suelo cal, suelo asfalto y otros productos diversos (DGIP, 2015). Además, los suelos arcillosos generalmente no cumplen con los requerimientos necesarios para garantizar la estabilidad del pavimento debido a su baja resistencia al corte, altas deformaciones e índice de plasticidad (Núñez \& Gil, 2018).

A la vez, si el terreno existente conocido como subrasante no asegura la estabilidad y durabilidad que garantice geotécnicamente el comportamiento de la estructura del pavimento se tiene dos opciones, el de sustituir realizando grandes movimientos de tierra y la segunda opción es el de mejorar el suelo existente (Leiva, 2016).

Asimismo, los suelos inestables pueden crear problemas significativos en la estructura del pavimento, por tal motivo desde hace algunas décadas se ha tratado de realizar el mejoramiento de estos suelos empleando diversas técnicas de estabilización como la cal, cemento, productos asfalticos, cloruro de sodio, entre otros, y que se emplean de acuerdo a las características del suelo y el alcance de la vía (MTC, 2014), en la presente tesis se investigó un nuevo método para la estabilización de suelo arcilloso que consiste en el uso de bolsas de polietileno fundido donde se busca mejorar las propiedades físico-mecánicas del suelo de fundación.

Por otra parte, en la actualidad la contaminación ambiental es generada en gran medida es por la generación de residuos sólidos los cuales no se pueden biodegradar rápidamente y están contaminando el medio ambiente en gran medida, por tal motivo se trata de reciclar y dándole un mejor uso en mejoramiento de suelos (Leiva, 2016).

Por ejemplo, la generación total de residuos sólidos municipales para el distrito de Chachapoyas está estimada en $12.78 \mathrm{Tn}$ /día para el año 2018, esta generación total está conformada por la suma de la generación de los residuos sólidos domiciliarios que equivale a $10.98 \mathrm{Tn} /$ día, mientras que la generación de los residuos sólidos no domiciliarios equivale a $1.80 \mathrm{Tn} /$ día. Con respecto a la composición física de los residuos sólidos domiciliarios la fracción de materia orgánica ocupa el primer lugar y equivale al $69.13 \%$, seguido de los residuos sanitarios con $10.04 \%$, las bolsas de polietileno con $4.70 \%$, seguido del cartón que representa $2.41 \%$, luego el vidrio el
$2.21 \%$, el papel con $2.04 \%$ y otros $9.47 \%$. Actualmente la basura generada se arroja en botaderos a cielo abierto y algunas fuentes de agua; causando un grave daño ambiental ya que muchos de los residuos generados por las industrias y los hogares se demoran más de 50 años en ser descompuestos (Municipalidad Provincial de Chachapoyas, 2018).

Debido a esta problemática se plantea una pregunta a resolver ¿En qué medida las bolsas de polietileno influyen en el mejoramiento de un suelo arcilloso a nivel de subrasante? y se va comprobar si las bolsas de polietileno influyen o no en el mejoramiento de un suelo arcilloso a nivel de subrasante.

Por último la finalidad de esta investigación fue determinar la influencia de las bolsas de polietileno fundido en la estabilización de un suelo arcilloso a nivel de subrasante, también consiste en delimitar la zona de estudio en el Pueblo Joven 16 de Octubre Chachapoyas, así como obtener muestras del material en estudio para ser analizadas en el laboratorio para determinar las propiedades físicomecánicas de las muestras en estudio antes y después de la adición de bolsas de polietileno fundido y estimar estadísticamente la proporción adecuada de bolsas de polietileno fundido que respondan a las condiciones impuestas en la norma técnica peruana.

\section{MATERIALES Y MÉTODOS}

\section{Localización}

El día 30/04/19 a las 8.45 am se extrajo $55 \mathrm{~kg}$ de material arcilloso por repetición para su análisis de laboratorio, fue de la calicata realizada en la intersección de la Av. Los Libertadores y Jr. Las Orquídeas, Pueblo Joven 16 de Octubre, Chachapoyas, Amazonas, Perú.

\section{Diseño de la investigación}

La investigación es de tipo experimental y se aplicó el Diseño Completamente al Azar (DCA).

\section{Método Inductivo}

Se inicia con la observación de casos particulares (toma de muestras de suelo) a casos generales (conocer su propiedades físicas y mecánicas), que se aplicaron en el laboratorio con adición de bolsas de polietileno fundido para el mejoramiento del suelo a nivel de la subrasante de la intersección de la Av. Los Libertadores y Jr. Las Orquídeas, Pueblo Joven 16 de Octubre, Chachapoyas.

\section{Metodología y procedimientos}

Para esta investigación se utilizó el método estadístico Diseño Completamente al Azar (DCA), específicamente la prueba de comparaciones múltiples Diferencia Mínima Significativa (DMS) que consiste en identificar el tratamiento $(0 \%, 4 \%$, 
8\% y 12\%) que presentó mayor incidencia.

Para cumplir con los objetivos planteados en la investigación, el estudio se realizó en tres fases:

\section{Fase 1: Fase preliminar}

\section{- Localizar y ubicar la muestra}

Se hizo un recorrido por el Pueblo Joven 16 de Octubre y se seleccionó el lugar donde se observó la presencia de suelo arcilloso (Intersección de la Av. Los Libertadores y Jr. Las Orquídeas).

\section{- Trabajo de campo}

La obtención de las muestras para el estudio de mecánica de suelos, se realizó en el Pueblo Joven 16 de Octubre (intersección de la Av. Los Libertadores y Jr. Las Orquídeas), Chachapoyas.

En el registro de excavación se anotó el espesor de los estratos del suelo; las muestras disturbadas representativas de suelos, se han obtenido en cantidades suficientes para realizar las cinco repeticiones de los ensayos de laboratorio, habiendo sido debidamente identificadas y embaladas en sacos y bolsas plásticas de polietileno para su conservación y traslado al laboratorio.

La excavación se ejecutó con herramientas manuales como son pico, barreta y palana, posteriormente se almacenó las muestras en sacos y bolsas para luego ser transportadas al laboratorio.

Se excavó una profundidad de 1.70 metros, se extrajo muestras de los tres estratos inferiores al material orgánico para ser analizadas en laboratorio, donde se observó la presencia de arena limosas en los estratos M1 y M2 y arcilla en el estrato M3 de la calicata.

\section{- Reciclaje de bolsas de polietileno}

\section{Identificación del botadero}

Para esta investigación se seleccionó el Botadero Rondón de Chachapoyas.

\section{Reciclaje de bolsas de polietileno en el botadero} Se reciclaron las bolsas de cualquier código de identificación de plástico, la cual señala la composición de los plásticos elaborada por la sociedad de la industria de plásticos.

\section{Procesamiento de las bolsas de polietileno y uso}

Se realizó el fundido de las bolsas de polietileno en una máquina cacera que consta de dos latas de aceite vacías que están conectado entre sí mediante un tubo galvanizado para no contaminar el medio ambiente, en una de las latas se encuentra bolsas de polietileno y la otra con agua para atrapar el humo cuando se realiza la combustión. El líquido producto de la fundición se mezcló con el material del suelo arcilloso en la proporción 1:1 (un litro de bolsas de polietileno fundido y un kilo de material arcilloso). El agregado base constituido, sirvió para elaborar las muestras tomando proporciones de $0 \%, 4 \%, 8 \%$ y
$12 \%$ respecto al peso seco de la muestra.

\section{Fase 2: Fase experimental}

- Ensayos para determinar las propiedades físicas-mecánicas.

\section{Propiedades físicas}

-Límite líquido - MTC E 110

-Límite plástico - MTC E 111

-Análisis granulométrico por tamizado - MTC E 107

-Clasificación de suelos método SUCS - NTP 339.134

-Clasificación de suelos método AASHTO - NTP 339.135

\section{Propiedades mecánicas}

-Proctor modificado - MTC E 115

-California Bearing Ratio (CBR) - MTC E 132

\section{- Tratamiento del suelo arcilloso}

La muestra base (suelo más bolsa fundida) fue elaborada de la siguiente manera, se fundió las bolsas de polietileno, el líquido producto de la fundición se mezcló con el material del suelo arcilloso en la proporción 1:1 (un litro de bolsas de polietileno fundido y un kilo de material arcilloso). El agregado base constituido, sirvió para elaborar las muestras tomando proporciones de $0 \%, 4 \%, 8 \%$ y $12 \%$ respecto al peso seco de la muestra.

\section{Fase 3: Fase de gabinete}

\section{- Procesamiento y análisis de datos}

Para esta investigación se procesaron los datos con el programa Microsoft Excel, y también analizamos los tratamientos propuestos con el método estadístico Diseño Completamente al Azar (DCA) específicamente la prueba de comparaciones múltiples Diferencia Mínima Significativa(DMS). 


\section{RESULTADOS}

Tabla 1. Resultados de ensayos de las cinco repeticiones

\begin{tabular}{|c|c|c|c|c|c|c|c|c|c|}
\hline ADICIONES & REP. & $\begin{array}{l}\mathrm{LL} \\
(\%)\end{array}$ & $\begin{array}{l}\text { LP } \\
(\%)\end{array}$ & $\begin{array}{c}\text { IP } \\
(\%)\end{array}$ & SUCS & ASSHTO & $\begin{array}{c}\mathrm{MDS} \\
\left(\mathrm{Kg} / \mathrm{cm}^{3}\right)\end{array}$ & $\begin{array}{c}\mathrm{OCH} \\
(\%)\end{array}$ & $\begin{array}{c}\text { CBR } 95 \\
\%\end{array}$ \\
\hline \multirow{5}{*}{$0 \% \mathrm{BPF}$} & 1 & 39.5 & 25.87 & 13.63 & $\mathrm{CL}$ & A-6(9) & 1.806 & 19.07 & 5.9 \\
\hline & 2 & 39.6 & 26.9 & 12.7 & ML & A-6(7) & 1.715 & 18.1 & 5.6 \\
\hline & 3 & 40.5 & 27.5 & 13 & ML & A-7-6(9) & 1.713 & 18.3 & 5.6 \\
\hline & 4 & 38.6 & 23.9 & 14.7 & $\mathrm{CL}$ & A-6(9) & 1.707 & 17.9 & 5.7 \\
\hline & 5 & 38.4 & 24.7 & 13.7 & $\mathrm{CL}$ & A-6(5) & 1.711 & 17.8 & 5 \\
\hline \multirow{5}{*}{$4 \% \mathrm{BPF}$} & 1 & 33.8 & 25.4 & 8.4 & $\mathrm{CL}$ & A-4(4) & 1.817 & 16.13 & 7.5 \\
\hline & 2 & 34.6 & 27.1 & 7.5 & $\mathrm{ML}$ & A-4(4) & 1.739 & 16.2 & 7.1 \\
\hline & 3 & 34.9 & 26.7 & 8.2 & ML & $A-4(3)$ & 1.741 & 16.4 & 7.3 \\
\hline & 4 & 33.7 & 23.4 & 10.3 & $\mathrm{CL}$ & $A-6(3)$ & 1.737 & 16.3 & 7.1 \\
\hline & 5 & 34.2 & 23.7 & 10.5 & $\mathrm{CL}$ & $A-6(3)$ & 1.731 & 16.6 & 6.9 \\
\hline \multirow{5}{*}{$8 \% \mathrm{BPF}$} & 1 & 35.9 & 22.6 & 13.3 & $\mathrm{CL}$ & A-6(6) & 1.823 & 16.92 & 8.9 \\
\hline & 2 & 35.4 & 23.6 & 11.8 & $\mathrm{CL}$ & A-6(5) & 1.783 & 15.8 & 9.5 \\
\hline & 3 & 36 & 24 & 12 & $\mathrm{CL}$ & A-6(6) & 1.773 & 15.5 & 9.3 \\
\hline & 4 & 35.5 & 24 & 11.5 & $\mathrm{CL}$ & A-6(3) & 1.785 & 15.2 & 9.6 \\
\hline & 5 & 36.3 & 24.2 & 12.1 & $\mathrm{CL}$ & A-6(3) & 1.783 & 15.4 & 9.5 \\
\hline \multirow{5}{*}{$12 \% \mathrm{BPF}$} & 1 & 39 & 25.36 & 13.64 & $\mathrm{CL}$ & A-6(7) & 1.833 & 16.68 & 9.8 \\
\hline & 2 & 37.8 & 23.7 & 14.1 & $\mathrm{CL}$ & A-6(7) & 1.793 & 15.3 & 9.9 \\
\hline & 3 & 38.9 & 25.1 & 13.8 & $\mathrm{CL}$ & A-6(7) & 1.785 & 15.2 & 9.8 \\
\hline & 4 & 37.5 & 24.6 & 12.9 & $\mathrm{CL}$ & A-6(4) & 1.801 & 15 & 9.9 \\
\hline & 5 & 37.7 & 23.9 & 13.8 & $\mathrm{CL}$ & A-6(4) & 1.823 & 15.1 & 10.3 \\
\hline
\end{tabular}

\section{ANÁLISIS ESTADÍSTICO}

A continuación, se presenta el análisis estadístico del CBR, propiedad más importante en el estudio de suelos ya que es la capacidad de soporte que presenta los suelos a resistir cargas.

\section{Análisis de varianza}

\section{Para la probabilidad}

- $\quad \mathrm{Si} \mathrm{Fc}<\mathrm{Ft} 5 \%$ y Ft 1\% $\Longrightarrow$ Nomenclatura(ns) $=$ No hay diferencia significativa entre las adiciones de bolsas de polietileno fundido.

- $\quad$ Si Ft 5\% $<$ Fc $<$ Ft 1\% $\Longrightarrow$ Nomenclatura $(*)$ $=$ Hay diferencia significativa entre las adiciones de bolsas de polietileno fundido.

- $\quad \mathrm{SiFc}>\mathrm{Ft} 5 \%$ y Ft 1\% $\Longrightarrow$ Nomenclatura (* $*)$ = Hay diferencia altamente significativa entre las adiciones de bolsas de polietileno fundido.

Para el coeficiente de variación $(\mathrm{CV})$

Cuando el $\mathrm{CV}<35 \%$, indica que los datos medidos en el experimento son confiables.
Cuando el $\mathrm{CV}>35 \%$, indica que los datos medidos en el experimento no son confiables.

California Bearing Ratio (CBR)

$\mathrm{N}^{\circ}$ de repeticiones $=5$

$\mathrm{N}^{\circ}$ de combinaciones $=4$

$\mathrm{N}^{\circ}$ de observaciones $\quad=20$

Factor de corrección $\quad=1283.202$

Coeficiente de variación $(\%)=3.34$

Tabla 2. Análisis de varianza para CBR

\begin{tabular}{|c|c|c|c|c|c|c|}
\hline $\mathrm{FV}$ & GL & $\mathrm{SC}$ & $\mathrm{CM}$ & Fc & $\begin{array}{c}\mathrm{Ft} \\
5 \%\end{array}$ & $\begin{array}{c}\mathrm{Ft} \\
1 \% \\
\end{array}$ \\
\hline TRAT. & 3 & 61.194 & 20.398 & \multirow{3}{*}{$\begin{array}{c}285.29 \\
(* *)\end{array}$} & \multirow{3}{*}{3.24} & \multirow{3}{*}{5.29} \\
\hline $\mathrm{EE}$ & 16 & 1.144 & 0.072 & & & \\
\hline TOTAL & 19 & 62.338 & & & & \\
\hline
\end{tabular}

Hipótesis nula $(\mathrm{Ho}): \mathrm{T} 0=\mathrm{T} 1=\mathrm{T} 2=\mathrm{T} 3$

Hipótesis alterna (Ha): Al menos 2 son diferentes

Como el $\mathrm{Fc}$ es mayor que el $\mathrm{Ft} 5 \%$ y el $\mathrm{Ft} 1 \%$ se rechaza la hipótesis nula, hay diferencia altamente 
significativa entre adiciones de bolsas de polietileno fundido.

\section{Prueba estadística diferencia mínima} significativa (DMS)

Como: Fc $>$ Ft 5\%, se rechaza la hipótesis nula, al rechazar la hipótesis nula podemos proceder a realizar la prueba de diferencias mínimas significativas.

\section{Planteamiento de la hipótesis}

Ho: $\mathrm{Ui}=\mathrm{Uj}$ No se establece la diferencia de las medias

$\mathrm{H} 1: \mathrm{Ui} \neq \mathrm{Uj}$ Las medias son diferentes

\section{Cálculo de parámetros}

$\mathrm{n}=5$

$\alpha=0.05$ (5\% Diferencias significativas)

$\mathrm{GLEE}=16$

$\mathrm{T}-$ Student $=1.7459$

DMS $=t(\alpha, G L E E) \sqrt{\frac{2 C M E E}{n}}$

DMS $=(1.7459) \sqrt{\frac{2(0.072)}{5}}$

\section{Criterios de rechazo}

$|\mathrm{Yi}-\mathrm{Yj}| \leq \mathrm{DMS}$ No se rechaza la hipótesis nula

$|Y i-Y j| \geq D M S$ Se rechaza la hipótesis nula

Tabla 3. Promedio de tratamientos para CBR

\begin{tabular}{|c|c|c|c|c|c|}
\hline \multirow{2}{*}{ Ensayo } & \multirow{2}{*}{ Repetic. } & \multicolumn{4}{|c|}{$\begin{array}{c}\text { Tratamientos o adiciones } \\
\text { aplicadas }\end{array}$} \\
\hline & & $\mathrm{T}_{0}$ & $\mathrm{~T}_{1}$ & $\mathrm{~T}_{2}$ & $\mathrm{~T}_{3}$ \\
\hline \multirow{5}{*}{$\begin{array}{c}\text { California } \\
\text { Bearing } \\
\text { Ratio } \\
\text { (CBR) }\end{array}$} & 1 & 5.9 & 7.5 & 8.9 & 9.8 \\
\hline & 2 & 5.6 & 7.1 & 9.5 & 9.9 \\
\hline & 3 & 5.6 & 7.3 & 9.3 & 9.8 \\
\hline & 4 & 5.7 & 7.1 & 9.6 & 9.9 \\
\hline & 5 & 5 & 6.9 & 9.5 & 10.3 \\
\hline \multicolumn{2}{|c|}{$\begin{array}{l}\text { Medias de los } \\
\text { tratamientos }\end{array}$} & 5.6 & 7.2 & 9.4 & 9.9 \\
\hline
\end{tabular}

Leyenda:

$$
\begin{gathered}
0 \%=\mathrm{T} 0 \\
4 \%=\mathrm{T} 1 \\
8 \%=\mathrm{T} 2 \\
12 \%=\mathrm{T} 3
\end{gathered}
$$

\begin{tabular}{|c|c|c|c|}
\hline $\begin{array}{l}\text { Diferencia } \\
\text { de niveles }\end{array}$ & $\begin{array}{l}\text { Diferencia } \\
\text { de las } \\
\text { medias }\end{array}$ & Resultados & Conclusión \\
\hline $\mathrm{T}_{3}-\mathrm{T}_{2}$ & 0.5 & $\begin{array}{l}0.5> \\
0.296\end{array}$ & $\mathrm{~T}_{3} \neq \mathrm{T}_{2}$ \\
\hline $\mathrm{T}_{3}-\mathrm{T}_{1}$ & 2.7 & $\begin{array}{l}2.7> \\
0.296\end{array}$ & $\mathrm{~T}_{3} \neq \mathrm{T}_{1}$ \\
\hline $\mathrm{T}_{3}-\mathrm{T}_{0}$ & 4.3 & $\begin{array}{l}4.3> \\
0.296\end{array}$ & $\mathrm{~T}_{3} \neq \mathrm{T}_{0}$ \\
\hline $\mathrm{T}_{2}-\mathrm{T}_{1}$ & 2.2 & $\begin{array}{l}2.2> \\
0.296\end{array}$ & $\mathrm{~T}_{2} \neq \mathrm{T}_{1}$ \\
\hline $\mathrm{T}_{2}-\mathrm{T}_{0}$ & 3.8 & $\begin{array}{l}3.8> \\
0.296\end{array}$ & $\mathrm{~T}_{2} \neq \mathrm{T}_{0}$ \\
\hline $\mathrm{T}_{1}-\mathrm{T}_{0}$ & 1.6 & $\begin{array}{c}1.6> \\
0.296\end{array}$ & $\mathrm{~T}_{1} \neq \mathrm{T}_{0}$ \\
\hline
\end{tabular}

Orden de mayor a menor $(\rightarrow)$

$\begin{array}{cccc}\text { T3 } & \text { T2 } & \text { T1 } & \text { T0 } \\ 9.9 & 9.4 & 7.2 & 5.6\end{array}$

Tabla 4. Combinación de tratamientos para el CBR
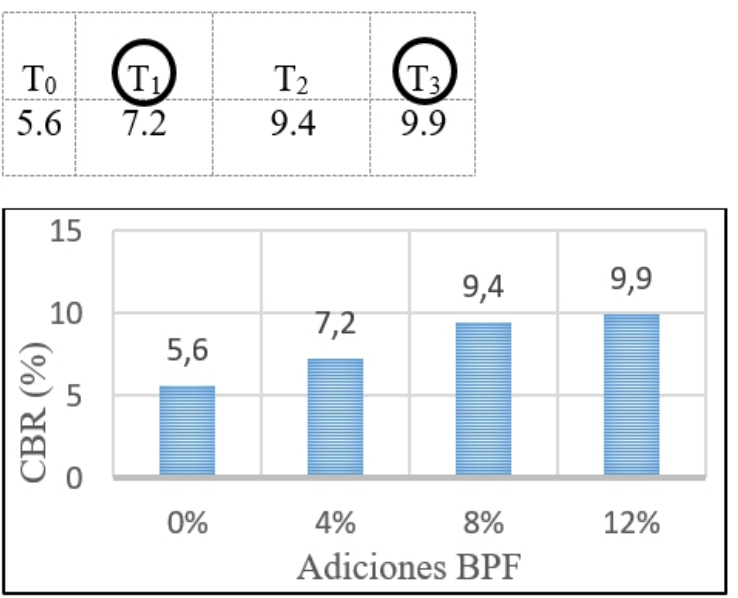

Todos los tratamientos se comportan de diferente manera, donde el tratamiento $12 \%$ (T3) tiene mejor incidencia en el CBR del suelo en estudio y el tratamiento 4\% (T1) es el que presenta menor incidencia.

Tabla 5. Resultados del análisis de varianza y prueba estadística diferencia mínima significativa(DMS) 


\begin{tabular}{|c|c|c|c|c|}
\hline \multirow{3}{*}{ Variables } & \multicolumn{3}{|c|}{ Análisis de varianza } & \multirow{3}{*}{$\begin{array}{c}\text { Diferencia } \\
\text { Mínima } \\
\text { Significativa }\end{array}$} \\
\hline & \multirow[t]{2}{*}{$\mathrm{Fc}$} & Ft & Ft & \\
\hline & & & $1 \%$ & \\
\hline \multirow{6}{*}{$\begin{array}{l}\text { Limite } \\
\text { líquido } \\
\text { (LL) }\end{array}$} & \multirow{6}{*}{$\begin{array}{l}64.6 \\
(* *)\end{array}$} & \multirow{6}{*}{3.24} & \multirow{6}{*}{5.29} & Mayor \\
\hline & & & & incidencia 4\% \\
\hline & & & & $\mathrm{T}_{1}=34.24 \%$ \\
\hline & & & & Menor \\
\hline & & & & $12 \%$ \\
\hline & & & & $\mathrm{T}_{3}=38.18 \%$ \\
\hline \multirow{7}{*}{$\begin{array}{l}\text { Limite } \\
\text { plástico } \\
\text { (LP) }\end{array}$} & \multirow{7}{*}{$\begin{array}{l}2.76 \\
\text { (ns) }\end{array}$} & \multirow{7}{*}{3.24} & \multirow{7}{*}{5.29} & Todos los \\
\hline & & & & tratamientos \\
\hline & & & & $4 \%\left(\mathrm{~T}_{1}\right), 8 \%$ \\
\hline & & & & $\left(\mathrm{T}_{2}\right)$ y $12 \%$ \\
\hline & & & & $\left(\mathrm{T}_{3}\right) \mathrm{se}$ \\
\hline & & & & comportan de \\
\hline & & & & $\begin{array}{l}\text { la misma } \\
\text { manera }\end{array}$ \\
\hline \multirow{7}{*}{$\begin{array}{l}\text { Índice } \\
\text { plástico } \\
\text { (IP) }\end{array}$} & \multirow{7}{*}{$\begin{array}{c}30.92 \\
(* *)\end{array}$} & \multirow{7}{*}{3.24} & \multirow{7}{*}{5.29} & Mayor \\
\hline & & & & incidencia $4 \%$ \\
\hline & & & & $\mathrm{T}_{1}=8.98 \%$ \\
\hline & & & & Menor \\
\hline & & & & incidencia \\
\hline & & & & $12 \%$ \\
\hline & & & & $\mathrm{T}_{3}=13.65 \%$ \\
\hline \multirow{9}{*}{$\begin{array}{c}\text { Máxima } \\
\text { densidad } \\
\text { seca } \\
(\mathrm{MDS})\end{array}$} & \multirow{9}{*}{$\begin{array}{l}6.20 \\
(* *)\end{array}$} & \multirow{9}{*}{3.24} & \multirow{9}{*}{5.29} & Mayor \\
\hline & & & & incidencia $8 \%$ \\
\hline & & & & $\left(\mathrm{T}_{2}\right)$ y $12 \%$ \\
\hline & & & & $\left(\mathrm{T}_{3}\right)=(1.789-$ \\
\hline & & & & $1.807) \mathrm{gr} / \mathrm{cm}^{3}$ \\
\hline & & & & Menor \\
\hline & & & & incidencia $4 \%$ \\
\hline & & & & $\mathrm{T}_{1}=1.753$ \\
\hline & & & & $\mathrm{gr} / \mathrm{cm}^{3}$ \\
\hline \multirow{7}{*}{$\begin{array}{l}\text { Óptimo } \\
\text { contenido } \\
\text { de } \\
\text { humedad } \\
(\mathrm{OCH})\end{array}$} & \multirow{7}{*}{$\begin{array}{c}25.15 \\
(* *)\end{array}$} & \multirow{7}{*}{3.24} & \multirow{7}{*}{5.29} & Mayor \\
\hline & & & & incidencia \\
\hline & & & & $12 \%$ \\
\hline & & & & $\mathrm{T}_{3}=15.46 \%$ \\
\hline & & & & Menor \\
\hline & & & & incidencia $4 \%$ \\
\hline & & & & $\mathrm{T}_{1}=16.33 \%$ \\
\hline & & & & Mayor \\
\hline & & & & incidencia \\
\hline California & 285.2 & & & $12 \%$ \\
\hline Bearing & 9 & 3.24 & 5.29 & $\mathrm{~T}_{3}=9.9 \%$ \\
\hline (CBR) & $(* *)$ & & & Menor \\
\hline & & & & incidencia $4 \%$ \\
\hline & & & & $\mathrm{T}_{1}=7.2 \%$ \\
\hline
\end{tabular}

\section{DISCUSIÓN}

Mediante el estudio realizado, se acepta la hipótesis planteada donde constituye que la adición de bolsas de polietileno fundido influye en el mejoramiento de un suelo arcilloso a nivel de subrasante, puesto que el índice de plasticidad disminuye y el CBR tiende a aumentar mediante la adición de bolsas de polietileno fundido.

La presente investigación presenta diferencia con lo que sostiene (Ramos, 2014), donde estudia el mismo material arcilloso con mediana plasticidad, pero observa que el CBR aumenta hasta un cierto porcentaje de adición de polímeros reciclados (PR) luego tiende a disminuir caso contrario sucede en la presente investigación ya que el valor del CBR aumenta a medida que se adiciona el porcentaje de bolsas de polietileno fundido.

La presente tesis guarda relación con lo expuesto por (Leiva, 2016) para sus propiedades mecánicas donde concluye que la adición de bolsas de polietileno en forma de grumos logra un aumento promedio del CBR de $7.98 \%$, con un tratamiento de $6 \%$ y en los resultados obtenidos en la presente investigación se observa que presenta un aumento promedio del CBR de $9.8 \%$ con el tratamiento $12 \%$. Al mismo tiempo presenta diferencia en sus propiedades físicas (Leiva, 2016) demuestra que a medida que se adiciona el porcentaje de aditivo el índice de plasticidad disminuye, caso contrario sucede en la presente investigación se observa que a medida que se adiciona el porcentaje de BPF el índice de plasticidad aumenta.

Se encuentra semejanza entre la presente investigación y lo que sostiene (López, 2013), donde ha demostrado que a medida que la cantidad de porcentaje de fibra de plástico reciclado (PET) aumenta, la resistencia del suelo también aumenta, en la presente investigación también sucede lo mismo ya que el CBR aumenta a medida que se adiciona el porcentaje de bolsas de polietileno fundido (BPF). Pero no pasa lo mismo con lo que demuestra (Leiva, 2016) y (Núñez \& Gil, 2018) ya que si aumentamos más aditivo el CBR tiende a disminuir.

El presente trabajo tiene relación con la investigación propuesta por (Núñez \& Gil, 2018) en la cual demuestra que los parámetros de compactación del suelo y a partir de mezclas de suelo arcilloso con fibras de PET reciclado variando el porcentaje de adición al $0.3 \%, 0.6 \%, 0.9 \%, 1.2 \%$ y $1.5 \%$ del peso seco del suelo. La máxima densidad seca del suelo varia mínimamente conforme aumenta la dosificación de la fibra, caso similar sucede en la presente investigación ya que si adicionamos más el porcentaje de bolsas de polietileno fundido mejor comportamiento la máxima densidad seca también aumenta.

Se encuentra diferencia entre la presente investigación y lo que sostiene (Cuipal, 2018) donde demuestra que con el menor porcentaje de adición de polímero sintético logra una mejor incidencia en las propiedades mecánicas del suelo, caso contrario sucede en la presente investigación ya que es el 
mayor porcentaje de adición de bolsas de polietileno fundido quien presenta mejor incidencia en las propiedades mecánicas del suelo.

La presente investigación resulta más económica en relación con lo que propone (Laurente, 2011) al añadir cal a la subrasante el costo unitario por $\mathrm{m} 2 \mathrm{es}$ 11.48 soles, en cambio para el presente estudio al añadir bolsas de polietileno fundido a la subrasante del suelo arcilloso el costo unitario por $\mathrm{m} 2$ es 6.46 soles.

\section{CONCLUSIONES}

- La adición de bolsas de polietileno fundido en proporciones de 4\%, $8 \%$ y $12 \%$ influyó en el mejoramiento de un suelo arcilloso a nivel de subrasante, por lo tanto, se acierta la hipótesis planteada donde establece que las bolsas de polietileno fundido influyen en el mejoramiento de un suelo arcilloso a nivel de subrasante.

- Se determinó las propiedades físicas y mecánicas de las muestras del suelo natural y del suelo natural más adición de bolsas de polietileno fundido, para las propiedades físicas el suelo según AASHTO varía de A-4(3) a A-7-6(9), asimismo según SUCS varía de de ML a CL; el índice de plasticidad disminuye de $13.55 \%$ (suelo natural) hasta $8.98 \%$ (4\% BPF); para las propiedades mecánicas el óptimo contenido de humedad tiende a disminuir mientras que la máxima densidad seca aumenta a medida que se incrementa los porcentajes de BPF y el CBR varía de 5\% a 5.9\% lo cual indica que la subrasante es pobre en resistencia y necesita ser mejorada; y para el suelo natural más adición del $12 \%$ BPF el CBR varia $9.8 \%$ a $10.3 \%$ lo cual indica que la subrasante es regular o buena. Por lo tanto, se logró estabilizar el suelo en estudio según del Manual de Carreteras Suelos, Geología, Geotecnia y Pavimentos ya que al añadir 12\% BPF el CBR es mayor que $6 \%$.

- En relación con la prueba estadística de comparaciones múltiples Diferencia Mínima Significativa (DMS), se determinó que para las propiedades físicas el tratamiento que presentó mejor incidencia fue el T1 (4\%) y para las propiedades mecánicas del suelo el tratamiento que presentó mejor incidencia fue el T3 (12\%).

\section{REFERENCIAS BIBLIOGRÁFICAS}

Blázquez, B. (2000). Curva Granulométrica de un Suelo.

CAPECO. (2019). Camara Peruana de la Construcción. Federación de trabajadores en construcción civil del Peru. Tabla salarial con beneficios sociales. Lima.
Cuipal, B. K. (2018). Estabilización de la subrasante de suelo arcilloso con uso de polímero sintético en la carretera Chachapoyas Huancas, Amazonas, 2018. (Tesis para obtener el Título Profesioanal de Ingeniero Civil) de la UCV. Perú.

DGIP. (2015). Dirección General de Inversión Pública. Pautas metodológicas para el desarrollo de alternativas de pavimentos en la formulación y evaluación. Perú.

ICG. (2000). CBR del suelo. Laboratorio MTC E 132. Lima.

Juárez, E. (2005). Mecánica de suelos tomo I. Fundamentos de la Mecánica de Suelos. México.

Laurente, Y. (2011). Estudio comparativo del mejoramiento de la subrasante y base de la carretera Cañete-Chupaca, Tramo: Km $220+000-K m 240+000$. (Tesis para obtener el Título Profesioanal de Ingeniero Civil) de la UNI. Lima.

Leiva, R. (2016). Utilización de bolsas de polietileno para el mejoramiento de suelo a nivel de subrasante en el jr. Arequipa, progresiva km $0+000-k m 0+100$. (Tesis para obtener el Título Profesioanal de Ingeniero Civil) de la UNCP. Distrito de Orcotuna, Concepción. Huancayo.

López, J. (2013). Suelos arcillosos reforzados con materiales de plástico reciclado (PET). (Tesis para obtener el Título Profesioanal de Ingeniero Civil) de la Escuela de Ingeniería de Antoquia. Colombia.

MTC. (2014). Ministerio de Transportes y Comunicaciones, Manual de carreteras, Suelos, geología, geotecnia y pavimentos. Sección suelos y pavimentos. Perú.

MTC. (2014). Ministerio de Trasportes y Comunicaciones. Manual de Carreteras Conservación Vial. Perú.

MTC. (2016). Ministerio de Transportes y Comunicaciones. Manual de ensayo de materiales. Perú.

Municipalidad Provincial de Chachapoyas. (2013). Plan de desarrollo urbano de la Cuidad de Chachapoyas. Volumen I y II. Chachapoyas.

Municipalidad Provincial de Chachapoyas. (2018). Gerencia Municipal. Unidad de Residuos Sólidos. Estudio de Caracterización de 
Residuos Sólidos Municipales. Chachapoyas.

Núñez, I. K., \& Gil, E. R. (2018). Influencia de la adición de fibras de PET reciclado sobre la resistencia, cohesión y ángulo de fricción interna de suelos arcillosos aplicado a la estabilizacion de taludes. (Tesis para obtener el Título Profesioanal de Ingeniero Civil) de la UNT. Perú.

OEFA. (2014). Organismo de Evaluación y Fiscalización Ambiental. Fiscalización ambiental en residuos sólidos de gestión municipal provincial. Perú.

Ramos, G. (2014). Mejoramiento de sub-rasantes de baja capacidad portante mediante el uso de polímeros reciclados en carreteras. (Tesis para obtener el Título Profesioanal de Ingeniero Civil) de la UNCP. Huancavelica.

RNE. (2010). Reglamento Nacional de Edificaciones. NORMA CE.010. Pavimentos Urbanos. Perú.

RNE. (2012). Reglamento Nacional de Edificaciones. NORMA CE.020. Estabilización de Suelos y Taludes. Perú.

Salazar, C. (2018). Fundamentos básicos de la estadística. Primera edición. Perú 